\title{
Making Active Learning Effective
}

\author{
John Pelley
}

Published online: 25 October 2014

(C) International Association of Medical Science Educators 2014

The question is not

"What is happening during our teaching?"

But,

"What is happening during their learning?"

The term "active learning" is a concept that is known more for what it is not. The emphasis on active learning originated as a contrast to the perceived "passive" atmosphere of the lecture. It quickly acquired a "face validity" since it is an instructional method that can be both seen and heard. However, a consideration of metacognition in this report will reveal that effectiveness is not assured for active learning, regardless of the appearance. These same insights also reveal that lectures do not have to be passive learning experiences. When the metacognition of active learning is truly understood, then its effectiveness will be assured and lectures could even regain some respect as an active learning environment.

Active learning in the medical curriculum usually involves the scheduling of small group activities to replace a portion of the lecture schedule. But, active learning has been defined more broadly to include all activities that are involved in information gathering and problem solving whether individual or with a group. For example, it has been proposed that active learning occurs as the result of the following tasks: (1) active construction of meaning (constructivism), (2) learning declarative knowledge (what?) and procedural knowledge (how?), (3) integration (transference between domains), (4) collaborative comparison of reasoning, and (5) meaningful learning through articulation [9]. Although these attempts to define and understand the process of active learning are comprehensive regarding pedagogical principles, the one element

J. Pelley $(\bowtie)$

Texas Tech University Health Sciences Center, Lubbock, TX, USA

e-mail: John.Pelley@ttuhsc.edu that determines effectiveness is still missing. That element is metacognition.

One of the three key findings of studies conducted by the Commission on Behavioral and Social Sciences and Education of the National Research Council revealed that the educational process can be hampered without an understanding of metacognition on the part of both students and teachers [3]. An understanding of the active learning process is essential to an understanding of effective teaching. Metacognition helps students take control of their own learning by defining goals and monitoring progress in achieving those goals. It also helps teachers to understand the responsibilities of the student in learning more than content. They must also learn how to learn. Lack of attention to metacognitive awareness results in an inefficient use of curriculum time by teachers and of learning time by students. By comparison, inclusion of metacognitive awareness facilitates the ultimate goal of medical education to assure transformation of the student into a self-directed, lifelong learner.

Metacognition is thinking about thinking. It is an examination of how information is processed and the factors both psychological and biological that contribute to learning from thinking. The use of metacognition is supported by research on the Growth Mindset, a belief that intelligence can be increased through hard work.

\section{Metacognition and Growth Mindset}

In order to develop a self-directed student, both the teacher and the student need to understand how the brain learns. Students who have this understanding demonstrate greater academic success as shown in studies that established the concept of the Growth Mindset [4]. Growth Mindset students 
believe that they can increase their intelligence through extra focused effort and motivation [2]. An informed teacher who understands how the brain learns can provide substantive support for students. Students that lack an awareness of how learning occurs simply robotically duplicate the teacher's portrayal of critical thinking and problem solving. If this were all that medical practice required, we could simply employ computers to deliver medical care. But, the patients seeking health care are part of a nonlinear complex world with their own chaotic and unpredictable outcomes [6]. Robotic thinking would lead to disaster.

The Growth Mindset prepares the students to assume responsibility for self-directed knowledge acquisition from their experiences. The process found to be most effective in acquiring expert skill from experience is known as Deliberate Practice [5]. It is likely that the emphasis on self-awareness in Deliberate Practice plays a role in the effectiveness of the Growth Mindset.

\section{Growth Mindset and Deliberate Practice}

The fundamental tenant of Deliberate Practice is for the student to develop a self-awareness that permits identification of weaknesses. This allows a teacher to help focus practice that can be repeated in order to improve a skill. For example, if a student has difficulty seeing relationships, the teacher can provide a list of related terms for the student to connect in a concept map. This approach to weakness requires, by its very nature, a motivation to do the practice needed. Without the guidance of a teacher, the more natural tendency is to practice what is more comfortable and less threatening. An example would be spending study time engaged in passive reading, a situation that provides comfort for many medical students by giving the impression that more material can be covered. A teacher who is not familiar with the Growth Mindset and the metacognitive correlates will mistake this reliance on rote memory for lack of intelligence.

An example of the Growth Mindset would be a student who reads deeply in order to construct a concept map, making continual decisions, and acting on their decisions to connect the related concepts even though this takes more time and is mentally tiring. Dweck attributes increased motivation to explain this extra effort [4]. The student who understands the superiority of Deliberate Practice believes that they can improve their own thinking skill and thus their own intelligence by working on their weakness. The improvement in thinking skill and intelligence is demonstrated with a student that has initial difficulty with concept mapping. Regular practice always produces greater speed and effectiveness over time, a reflection of increased thinking skill.

Since Deliberate Practice requires a teacher to guide the skill development during early stages, an effective role for the teacher would be to have the student verbalize their concept map of a topic allowing the teacher to confirm content and organization. By making the student's thinking visible, the concept map allows an opportunity for the teacher to reinforce correct thinking and to suggest improvements. An example of an improvement might be a suggestion to look more carefully for grouping concepts [see Inspectional Reading, 1] that would allow a branch point to connect related facts such as the symptoms of a heart attack with "heart attack" as the grouping term. This situation fulfills the characteristics of Deliberate Practice (concept mapping) while developing the Growth Mindset (understanding what concept mapping achieves). This can also be done with other study methods that the student might be using. Eventually, the student acquires enough skill that the teacher is no longer needed [5].

If Deliberate Practice is to be applied to learning, then its application to "learning style" must be known to both teachers and students. A student cannot understand the purpose of learning strategies if they do not understand how they learn. The Jungian preferences for taking in new information, the Sensing preference and the Intuitive preference, serve as useful and simple insights into differences in information processing. These preferences are also useful because they correspond to two major functional areas of the cortex and also to two major steps in the Experiential Learning Cycle [14].

\section{Deliberate Practice and Personality Type}

Personality type seems on the surface to have little to do with learning, much less to do with skill development. However, a review of the Jungian psychological types first described by Carl Jung in 1929 [7] and subsequently identified by the Myers-Briggs Type Indicator (MBTI), will show that not only is skill involved, but that multiple skills are involved [10]. The discussion of learning styles here will be limited to the Jungian mental processes that are derived from the psychological types because they match most closely with the functional specialization of the cortex and limbic system [12]. Although there are four pairs of opposites identified by the MBTI, only the Sensing/Intuitive pair will be considered here as the most relevant to learning style.

When the Sensing and Intuitive preferences are compared, they are seen as mutually exclusive opposites. If the comparison is limited only to a preference, then a powerful opportunity for skill development is missed. When viewed instead as intellectual skills, the mental processes that underlie the preference become mutually beneficial. Thus, the Sensing and Intuitive preferences can be referred to as a "learning style," and Sensing and Intuitive functions can be referred to as learning "skills" [12]. A preference is better understood as a comfort zone where the student spends most of their thinking time, but the student can apply Deliberate Practice to develop their opposite as a thinking skill. This is possible to some 
extent because all students adapt the use of both Sensing and intuition to each learning situation regardless of their preference.

A teacher can help the student to become aware of when and how to use their opposite and guide their development into a self-directed mentality. Everyone uses both "skills" while having only one "preference." [11]. Thus, a learning style is a preference not a limitation. An example is found in throwing a baseball. Throwing with the person's "handedness" is natural and done virtually unconsciously because motor function becomes automated over time. Switching hands to the "off-hand" also switches the attention of the person to the mechanics of throwing with the less used limb. The process is described by many as "child-like" and underdeveloped (clumsy). However, with practice, the off-hand can be improved in skill, and with a teacher aiding Deliberate Practice, this can happen at a more rapid pace. This is a very appropriate analogy to the cognitive handedness of personality type.

In the same way that an athlete or any other skilled performer can develop their "off-hand," a student can develop the less used components of their cognition. Thus, an Intuitive type would have the Sensing function as their off-hand and vice versa for the Intuitive type. Further, if an Intuitive type wanted to use Deliberate Practice to develop their Sensing skills, they would undertake learning activities that required active incorporation of detailed facts into the integration that they do naturally. An example is the addition of detailed facts to the tips of the branches in a concept map. Indeed, concept maps are excellent Deliberate Practice modalities because the Sensing-type student can improve their ability to discover integration and the Intuitive-type student can construct branches where they can hang all of those elusive details.

\section{Personality Type and Experiential Learning Cycle}

If skill development is to be applied to learning, then learning must be defined in terms of each skill required. Just as a tennis player must develop a serve, a backhand, and other skills in order to perform all possible phases of the game, a student must acquire the requisite skills to perform in all possible phases of decision-making during learning. Learning in medicine is fundamentally no different than learning in any performance venue, e.g., athletics, music, and chess. In every case, the performer's brain must convert sensory information into action and every action requires a decision. How each individual learns is influenced by the way they uniquely sense and recognize the world around them, how they create alternative responses, and how they act on a decision for one of those responses (including no response, which is a decision).

The Jungian Sensing types tend to use their brain in the present moment. The term "sensing" was chosen by Jung to represent a behavior that gives most of the attention to what is immediate, i.e., what can be sensed. By comparison, the Jungian Intuitive types give their attention to the future moment that contains alternative outcomes. The term "intuitive" was chosen by Jung to indicate a "sixth sense" that gave meaning to what was being experienced. To paraphrase, Sensing types ask "what is?" while Intuitive types ask "what if?" If either question is taken alone, thinking is incomplete and learning is ineffective. By reframing these preferences as skills, it can be argued that a Sensing type that has not developed the balancing Intuitive skill will be devoid of imagination and, worse, devoid of meaning. Their world would be one of dependency on recognition of stimuli and responding robotically - a description that also applies to a computer. On the other hand, the Intuitive type without the balancing Sensing skill will be missing essential facts needed to formulate reasonable integration of alternatives, e.g., forming a differential diagnosis. Their intuitive creativity would thus be rendered irrelevant. The only effective approach to the education of the medical professional is the development of skill in both the Sensing function and the Intuitive function regardless of the underlying preference. With sufficient motivation (from the Growth Mindset), this is readily accomplished through Deliberate Practice.

The Experiential Learning Cycle (ELC) described by Kolb [8] is a model derived from pedagogical theory that fully integrates the steps that produce learning from experience. It is composed of four repeating steps: (1) concrete experience, (2) reflective observation, (3) abstract conceptualization, and (4) active testing. The last step produces an outcome which provides new concrete experience that begins a new cycle. This mental model not only correlates information processing with the Jungian mental functions of Sensing and intuition, but it also correlates information processing with the functional specialization of the cortex [14]. The first step in the ELC is the processing of sensory information by areas of the cortex at the back of the brain so that it can be recognized (concrete experience). This applies to words on a page as readily as spots on an X-ray. The second step is also "rear brained" as the temporal area attempts to recognize the sensory input (reflective observation). Both of these areas work together to process information sensed in the present and retrieved from long term memory. This rear brain function matches closely to what is referred to above as Sensing skills. Thus Sensing types would tend to use this part of the brain to a greater extent than the frontal area. The third step is the integration of new sensory input (e.g., reading a chapter) with what has already been learned. This is accomplished through connections between the prefrontal cortex and the rear brain cortex (frontoparietal). The prefrontal area tries to create meaning by developing alternatives or relationships and then narrowing the list to make a decision. The fourth step is the conversion of the decision into action by an area in the frontal cortex called the motor area. Since the speech area, Broca's area, is also 
located in this region, action can be in the form of speaking, e.g., small group dialogue. However, speech alone does not guarantee active learning. If the dialogue is simply recited (a typical Sensing-type response), then the prefrontal area is bypassed and no integrative thinking has occurred. On the other hand, the front brain is engaged when the discussion requires a rationale. The formation of a rationale does require integrative thinking (a typical Intuitive-type response). Thus Intuitive types would tend to use future predictions and anticipation of cause-and-effect relationships in their thinking.

It becomes evident when all of the functional areas of the cortex are taken into account, that the development of skill in all four stages of the ELC is essential to development of higher order thinking skills. Likewise, a limitation in any one skill will limit the effectiveness of the remainder (Fig. 1). It is important to keep the emphasis on "skill," as opposed to "smart," because this helps to differentiate growth in intelligence from a fixed level of intelligence. While future studies may reveal ways of identifying more precisely deficits in the first and last skills in the ELC (sensory skill and motor skill), deficits in the second and third (long-term memory and analysis) are readily identified. As previously described, the second skill that recognizes and remembers explicit knowledge, i.e., facts and concepts, is less used by the Intuitive types and strategies for correcting this deficit should include utilizing important details in making decisions during study. It is always more effective to approach the development of the respective skill areas in the cortex through the preference, or learning style, of the student. As just mentioned for the Intuitive type, it is most effective to take advantage of their integrative abilities to integrate hard-to-learn facts. Likewise, the Sensing type that has difficulty in finding patterns and relationships will find that step-by-step guidelines will make this task approachable.

The ELC becomes less theoretical when it is correlated with cortical function, but even more so when it is explained at the cellular level. Each step in the ELC uses a corresponding

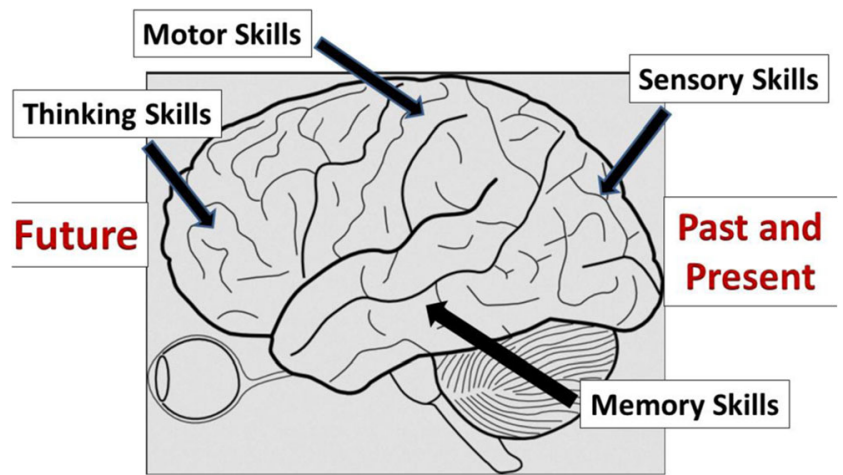

Fig. 1 The cerebral cortex viewed from the left. Skill areas referred to the text represent the functional organization of the cortex. The rear brain (sensory and memory) processes the past and present experience while the front brain (thinking and motor) anticipates the future. Copyright $(C)$ Tooloop.com - Free Anatomy Picture Source 2014 skill area in the cortex, and each skill area improves in function through changes at the cellular level during sleep. The ELC involves action, and action is a critical determinant of the learning events that occur during sleep.

\section{Experiential Learning Cycle and The importance of Action-Emotion and Sleep}

The last step in the ELC is the most critical step for producing long-term learning. This step requires a decision which produces action. Every action, in turn, is accompanied by an emotion as an additional outcome of the ELC. Thus, active learning is emotional learning. The emotion can be positive or negative, but it is the emotional component that drives the critical learning events during sleep.

During the day when we are conscious, the brain is continually forming new synaptic connections related to the activity of the ELC. Every area of the cortex that is used is undergoing a form of cellular growth, not from cell division but from nerve cell branching. Dendrites branch (Fig. 2) and grow small projections called spines where future synapses will form. All of this occurs as we think, decide, and act. This explains why we can sit and read for several hours and be able to think and talk about what we have been reading. However, this is not yet learning. It is an "illusion of learning" because the actual learning event occurs later during sleep.

During sleep, the brain fluctuates its arousal state (more accurately, susceptibility to arousal) in a 90-min sleep cycle. Arousal is more difficult during deep sleep compared to the more shallow rapid eye movement (REM) sleep when dreaming occurs. Each sleep cycle involves a replay of all previous events during the waking hours. Explicit memory, the memory for facts and concepts, is consolidated (made permanent) during slow wave, deep sleep with the maximum consolidation achieved after 5 cycles [13]. The consolidation event is

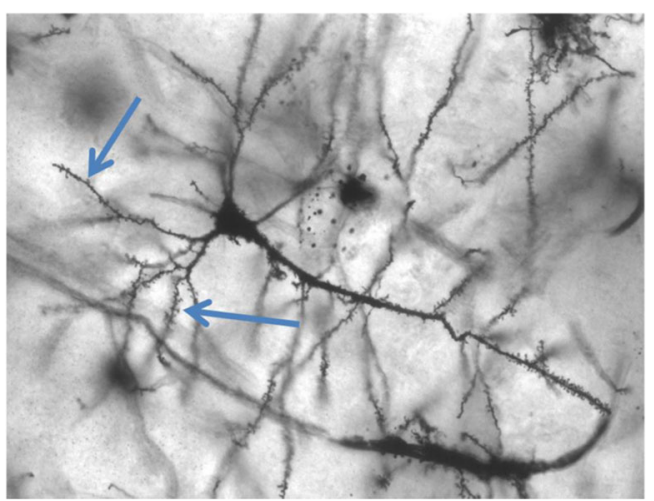

Fig. 2 Neurons stained selectively to reveal branching of dendrites. Small projections (arrows) from dendrites are spines (future synapses). Branching forms during daily learning experiences but is pruned away if not associated with emotion. Wikimedia Commons the free media repository 
dependent on emotion to either prune unwanted memories or to maintain and strengthen emotionally related useful memories. In other words, you cannot remember anything you do not care about.

Consolidation of strong synapses is readily measured for the back of the brain with testing of memorized knowledge. Depending on how retrospective the selection of topics tested, even the persistence of long-term memory can be estimated. This type of memory is processed in the temporal area of the rear cortex and Sensing types generally have a preference for using this skill as shown by a highly developed ability to remember facts and details. Consolidation in the front of the brain is observed differently, but an alert teacher will recognize it as an increase in problem solving and/or decisionmaking speed. When the student is engaged in active learning by discussing and deciding on alternatives, the synapses are consolidated in the prefrontal area to produce an increased computing capacity. The prefrontal area acquires the skill of retrieval from the back of the brain, creation of alternative meanings, and narrowing to select the most logical alternative. These processes use short-term (volatile) memory, but the goal is a decision not remembering. Taken together, the rear cortex and the prefrontal cortex process sensory input to produce both long-term memory and increased speed in accessing and processing that memory to produce action.

The crucial role of sleep in learning and the pivotal role of emotion in determining consolidation pose challenges for both the student and the teacher. The student will experience the most efficient learning with the maximum of activity (motor skills) during learning. Taken together, writing while speaking about the underlying thinking will produce a tangible product as well as the emotion needed to consolidate the learning. The teacher will experience the most efficient teaching with learning objectives that require more complex tasks such as comparisons (similarities and differences) and cause-and-effect relationships. The direction provided by learning objectives will help the student focus their Deliberate Practice in the most effective manner.

\section{Summary-Active Learning As Decision-Making}

Active learning cannot be effective without a metacognitive awareness by both the student and the teacher. This awareness enables self-directed learning decisions on the part of the student both during the group interactions and afterward during individual follow-up learning. Metacognitive awareness likewise enables teachers to better prepare the students to manage their group interactions so that they are able to make the best use of time following the active learning session. Examples would be teacher-generated learning objectives that emphasize both factual and integrative content and student attention to dialogue from group members with their opposite learning style during group sessions. If metacognition is left out of the teaching strategy, it is likely to be left out of the learning strategy.

Even the passive appearance of the lecture setting can be an active learning environment when metacognition is involved. A metacognitive student follows a disciplined protocol after a lecture aimed at reorganizing the content and extending it to integrate with past learning. This student listens differently while in the lecture than the student who merely receives the information passively. A self-aware student will conduct their individual learning time after lecture to direct their attention to their learning weakness so that difficult issues can be taken to a teacher for focused discussion. They will "listen for" potential post-lecture organizational concepts instead of "listening to" a stream of information to be memorized. A self-aware student, therefore, becomes increasingly self-directed regardless of the environment. Whether they are in lecture or a small group, or alone, they "listen for" and "look for" rather than "listening to" or "looking at." They have an awareness of their own learning and learning needs.

In this report, multiple interrelated concepts have been used to illustrate what metacognition is and how it should be employed. The Growth Mindset was considered first to bring the evidence that metacognition produces higher academic achievement. The Growth Mindset was then linked to Deliberate Practice to propose a means by which the student would develop the skills needed for this higher achievement. The validity of Deliberate Practice has been established as the only correlate for those who have achieved superior skill development. In order to expand the semantic of "learning skill," personality type as identified by the MBTI served as an example of how thinking can follow the same process and yet emphasize one step in that process over others. At this point, the basis for self-awareness also emerges and suggests how Deliberate Practice can be used to develop the skill (not the preference!) of the non-preferred process. The processes that were contrasted, Sensing (memorization oriented) and Intuition (integration oriented) provide examples seen in medical student learning on a regular basis. While these examples provide a basis for implementing Deliberate Practice for the student, a consideration of neurobiology and the role of sleep remains a further essential metacognitive insight. The profound correlation of pedagogical theory with the specialized function of each major cortical area highlights the major front/ rear roles in learning. The brain learns in order to create action from input. The interaction of recognition by the rear cortex and of decision by the frontal cortex can be conceived as an Experiential Learning Cycle. It is through this mental model that we understand the neurobiological meaning of active learning. We also learn how this model produces the consolidation events during sleep that are responsible for the enduring nature of long term learning.

Our current system of education neglects teaching "how to learn" as it maintains the focus on "what to learn." If we want 
to produce the best learning outcomes we must teach the student to be aware of how they learn and which of their learning skills are in need of strengthening. When metacognition is taken into account, a strategy is now possible for transforming the student into a self-directed and skilled learner. Metacognition creates control over learning for both the student and the teacher. The student assumes responsibility not only for creating their own understanding but for determining when the teacher is needed to correct weakness. The teacher assumes responsibility for orchestrating content and the learning objectives that bring that content to life in solving problems. Metacognition manages "causes" instead of "effects" and results in making active learning effective.

\section{References}

1. Adler M, Van Doren C. How to read a book. New York: Touchstone; 1972.

2. Atkinson JW, Feather NT. A theory of achievement motivation. Huntington: Robert E. Krieger Publishing Company; 1966.
3. Bransford JD, Brown AL, Cocking RR, editors. How people learn: brain, mind, experience, and school. Washington: National Academy Press; 2000.

4. Dweck C. Mindset: the new psychology of success. New York: Random House; 2006.

5. Ericsson KA. Deliberate practice and the acquisition and maintenance of expert performance in medicine and related domains. Acad Med. 2004;79(10 Suppl):S70-81.

6. Heng HHQ. The conflict between complex systems and reductionism. JAMA. 2008;300(13):1580-1.

7. Jung CG. Psychological types. Princeton, New Jersey: Princeton University Press; 1971.

8. Kolb DA. Experiential learning. Englewood Cliffs: Prentice Hall; 1984.

9. Michael J. Where's the evidence that active learning works? Adv Physiol Educ. 2006;30:159-67.

10. Myers IB, McCaulley MH, Quenk NL, Hammer AL. MBTI manual: a guide to the development and use of The Myers-Briggs Type Indicator. 3rd ed. Palo Alto: Consulting Psychologists Press; 1998.

11. Pelley JW, Dalley BK. Success types. In Medical education, www. ttuhsc.edu/SOM/success. 2008

12. Pelley JW. Learning style: implications for teaching and learning. In: Matheson D, editor. An introduction to the study of education. 4th ed. London: Routledge; 2014.

13. Stickgold R, Ellenbogen JM. 'Sleep on it: how snoozing makes you smarter', Scientific American Mind, August. (2008)

14. Zull J. The art of changing the brain. Sterling: Stylus Publishing, LLC.; 2002. 